\title{
Impacto psicológico da pandemia em gestantes e puérperas brasileiras
}

\author{
Psychological impact of the pandemic on brazilian \\ pregnant and postpartum women
}

\section{Alessandra Arrais', Bianca Amorim², Luciana Rocha ${ }^{3}$ e Ana Clara Haidar}

Resumo: Buscou-se entender o impacto psicológico em gestantes e puérperas brasileiras frente ao isolamento social e à pandemia de Covid-19. Realizou-se um estudo descritivo, transversal, exploratório, multicêntrico nacional, com 710 gestantes e 339 puérperas brasileiras. Aplicou-se um questionário on line sobre aspectos sociodemográficos e sentimentos relacionados à pandemia de Covid-19. Calcularam-se as frequências absoluta e relativa das respostas. As principais preocupações/temores encontradas foram: ter a Covid-19 e ser internada na UTI; o bebê precisar de UTIneonatal; ter a Covid-19 e perder o bebê, transmitir o coronavírus verticalmente para o bebê; o bebê adquirir uma má-formação se a gestante tiver a Covid-19, não ter acompanhante no parto ou pós-parto imediato e não poder amamentar o filho. Esses resultados motivaram a criação, pelas pesquisadoras, de um grupo voluntário formado por 21 psicólogos perinatais, o Grupo de Intervenção em Crise. Ele ofereceu atendimento on line à mulheres no ciclo gravídico-puerperal para fomentar estratégias para prevenir e mitigar o sofrimento delas durante o processo de gestar e tornar-se mãe, e os impactos negativos da pandemia da Covid-19 na sua saúde mental. Sugerimos a importância de estabelecer políticas urgentes focadas na saúde mental materna, especialmente durante a pandemia, dirigidas ou desenvolvidas por profissionais de saúde mental.

\section{Palavras-chave: Covid-19; Efeitos psicológicos; Gestantes.}

Abstract: We sought to understand the
psychological impact on Brazilian pregnant and
postpartum women in the face of social isolation
and the Covid-19 pandemic. A descriptive, cross-
sectional, exploratory, national multicenter study was
carried out, with 710 Brazilian pregnant women and
339 postpartum women. An online questionnaire on
sociodemographic aspects and feelings related to
the Covid-19 pandemic was applied. Absolute and

\footnotetext{
1 Pós-doutora pelo Programa de Pós-graduação em Psicologia Clínica e Cultura do Departamento de Psicologia da Universidade de Brasília (UnB). Docente permanente do Programa de Mestrado Profissional da Escola Superior de Ciências da Saúde (ESCS), Psicóloga da Secretaria de Estado de Saúde do Distrito Federal, Sócia-diretora e Professora da Escola de Profissionais da Parentalidade. E-mail: alearrais@gmail.com

2 Psicóloga (2007) pelo Centro Universitário de Brasília, pós-graduada em Psicanálise, Perinatalidade e Parentalidade pelo Instituto Gerar (2019). Psicoeducadora no projeto digital Renascendo após a maternidade, Psicóloga Clínica com foco perinatal e parental e Sócia-diretora e Professora da Escola de Profissionais da Parentalidade. Email: bianca@escoladaparentalidade.com.br

3 Psicóloga (2005) pela Universidade Católica de Brasília, pós-graduada em Psicologia Aplicada à saúde (2008) e pós-graduada em Psicanálise, Perinatalidade e Parentalidade pelo Instituto Gerar (2019). Psicóloga Clínica com foco perinatal e parental, idealizadora da fanpage Tons da Maternidade e Sócia-diretora e Professora da Escola de Profissionais da Parentalidade. Mestranda Programa de Pós-graduação em Psicologia Clínica e Cultura do Departamento de Psicologia da Universidade de Brasília (UnB). E-mail: luaroch@gmail.com

${ }^{4}$ Estatística da Universidade de Brasília (UnB). Membro da Empresa Júnior em Estatística - ESTAT Consultoria. Estagiária em estatística do Superior Tribunal de Justiça - STJ. E-mail: aninhaidar@gmail.com
} 
relative frequencies of responses were calculated. The main concerns/fears found were: having Covid-19 and being admitted to the ICU; the baby needs the NICU; having Covid-19 and losing the baby, transmitting the coronavirus vertically to the baby; the baby acquires a malformation if the pregnant woman has Covid-19, does not have a companion during the birth or immediate postpartum period and cannot breastfeed the child. These results motivated the creation, by the researchers, of a voluntary group formed by 21 perinatal psychologists, the Crisis Intervention Group.

\section{Introdução}

A Covid-19 é uma doença respiratória aguda, que apresenta uma taxa de mortalidade de cerca de 2\% (Fiocruz, 2020). Ela pode resultar em morte devido a danos importantes nos alvéolos pulmonares e insuficiência respiratória grave e progressiva (Huang et al. 2020; Xu, et al., 2020). A transmissão entre as pessoas se dá por meio de gotículas espalhadas pela tosse, espirro e fala de um indivíduo infectado, principalmente pelo contato direto ou pelo ar (Xu, et al., 2020). Por isso, o enfrentamento da Covid-19 preconiza lavar as mãos ou limpá-las comálcool em gel, frequentemente, evitar abraços, beijos e apertos de mãos, uso de máscaras em ambientes públicos e privados, e adotar medidas de distanciamento físico, de pelo menos dois metros de distância entre as pessoas, isolamento social e evitar aglomeração (Croda, \& Garcia, 2020). No Brasil, todas essas medidas foram parcialmente implementadas em todos os Estados, ainda que em momentos diferentes, na tentativa retardar e controlar a disseminaçãa do coronavírus entre as pessoas e prevenir a sobrecarga e colapso nos serviç̧os de saúde (Sarti et al. 2020; Croda, \& Garcia, 2020; Parmet, \& Sinha, 2020; Filho, \& Segall-Corrêa, 2020)

Não se sabe ao certo se o impacto de uma infeção por covid-19 poderá ser mais grave em uma grávida do que em outras pessoas. No entanto, com os vírus da mesma família do coronavirus, que causam outras infeções respiratórias virais, as gestantes apresentam maior risco de terem complicações, sendo a mais ameaçadora e rara delas, a Síndrome respiratória aguda grave (SRAG), que pode levar à morte (Favre et al., 2020). Em função dessas experiências anteriores, e após publicações mais recentes, a Organização Mundial da Saúde (WHO, 2019a) advertiu que as grávidas têm risco acrescido de contrair a infecção pelo coronavirus e passou a considerar que gestantes e puérperas também fazem parte do grupo de risco para o novo coronavírus (Takemoto et al., 2020; WHO, 2019b).

Logo começaram a ser registrados um aumento nos óbitos em gestantes, especialmente no Brasil, conforme estudo de Takemoto et al. (2020) que encontrou o registro de 978 casos de gestantes brasileiras diagnosticadas com Covid-19 até 18/06/20, sendo que 124 delas evoluíram para óbito (12,7\%). Esse estudo também revelou um dado grave, de que o Brasil é 0 ser o país com mais mortes maternas por Covid-19 no mundo. Na atualidade, essas mortes superam todas as mortes de gestantes somadas dos demais países.

Além dos impactos físicos tanto para as gestantes, cabe ressaltar também a importância de conhecermos o impacto da pandemia quanto à saúde mental materna (Fiocruz, 2020; Kang, 2020; Sahina, \& Kabakci, 2021; Puertas-Gonzalez et al., 2021). No caso das gestantes, parturientes e puérperas, acredita-se
It offered online care to women in the pregnancypuerperal cycle to promote strategies to prevent and mitigate their suffering during the process of pregnancy and becoming a mother, and the negative impacts of the Covid-19 pandemic on their mental health. We suggest the importance of establishing urgent policies focused on maternal mental health, especially during the pandemic, directed or developed by mental health professionals

Keywords: Covid-19; Psychological effects; Pregnant.

que esses estressores se sobrepõem e potencializam aqueles inerentes à gestação, parto e puerpério, potencializando a sobrecarga e o sofrimento psíquico dessa população (The Marcé Society, 2020; Puertas-Gonzalez et al., 2021; López-Morales et al., 2021). Em estudo recente, realizado por López-Morales et al. (2021), mulheres grávidas mostraram um aumento mais pronunciado de depressão, ansiedade e afeto negativo e diminuição mais pronunciada no afeto positivo do que mulheres não grávidas. Por outro lado, no estudo de Dong et al. (2021) com mulheres grávidas na China durante a epidemia de COVID-19, encontrou-se que apenas o nível de depressão era significativamente mais alto, enquanto o nível de ansiedade era mesmo de antes da epidemia. Mulheres grávidas que viviam em Wuhan, o epicentro da epidemia, não estavam mais ansiosas ou deprimidas em comparação com mulheres grávidas em outras regiões durante a epidemia de COVID-19. Além disso, o estado de saúde mental das mulheres grávidas com COVID-19 não era mais grave.

Além dessa sobrecarga emocional que o ciclo gravídico puerperal pode implicar, nos primeiros meses de 2020, as mulheres grávidas e puérperas também precisaram enfrentar a pandemia da Covid-19, incluindo as excepcionais medidas de quarentena que tanto perturbaram a vida privada e profissional da população mundial (Yang et al., 2020). Por tratar-se de um contexto recentee inesperado, estudos relacionados às implicações da pandemia pelo Covid-19 sobre a saúde mental da população em geral (Schmidt et al., 2020), mas especialmente daquela que se encontra no ciclo gravídico-puerperal, ainda é escasso (The Marcé Society, 2020).

Nesse sentido, este estudo se justifica, pois, tem o potencial de oferecer uma melhor compreensão dos sentimentos e comportamentos de gestantes durante a pandemia, bem como oferecer propostas de intervenção e reflexões para a prática profissional, em especial do psicólogo perinatal, dedicadas a atenuar o sofrimento psíquico dessa população nesse contexto pandêmico e 0 isolamento social (The Marcé Society, 2020). Considerando, portanto, a conjuntura atual, buscou-se entender o impacto psicológico em gestantes e puérperas brasileiras frente ao isolamento social e à pandemia de Covid-19

\section{Método}

Este artigo refere-se a um recorte da pesquisa-mãe, intitulada "0s Impactos Psicológicos da Pandemia em Gestantes e Mães de Bebês" e foi feita virtualmente com mulheres residentes nas cinco regiões do Brasil e tratou-se de uma pesquisa de opinião, sem identificação dos participantes, obedecendo às normas das Resoluções CNS/MS 466/2012 e 510/2016. 
0 presente recorte trata-se de estudo descritivo, transversal, exploratório, multicêntrico nacional. A amostra foi constituída por conveniência e contou com 1049 mulheres, sendo 710 gestantes e 339 puérperas recentes. Os critérios de inclusão foram: ter mais de 18 anos, ser capaz de responder o instrumento por meio de computadores ou smartphones e, no caso de puérperas, os bebês deveriam ter no máximo 60 dias de vida.

0 instrumento utilizado foi um questionário online construído pelas pesquisadoras por meio do Typeform ${ }^{\circledR}$. 0 questionário continha perguntas fechadas, de múltipla escolha e questões do tipo likert, na qual a participante deveria avaliar entre 1 e 5 o seu grau de preocupação/temores, considerando 1 "pouca preocupação/temor" 5 "extrema preocupação/temor"; e tratou sobre aspectos sociodemográficos (faixa etária, região de residência, estado civil, nível de escolaridade, ocupação, renda per capita); aspectos relacionados à pandemia pelo coronavírus (apresentação de sintomas, realização de testes, riscos de contágio, contato com pessoas infectadas, fonte das informações); e aspectos relacionados aos sentimentos de medos e de preocupações durante 0 isolamento social na pandemia (as gestantes foram questionadas sobre medos e preocupações em relação à gestação, ao parto e ao pós-parto e as puérperas foram perguntadas sobre temores, preocupações e comportamentos em relação ao pós-parto e ao bebê). Foi realizado um estudo piloto com cinco gestantes e cinco puérperas para adequação do instrumento quanto ao seu acesso digital, facilidade de manuseio, tempo de resposta e linguagem.

Para coleta de dados foram utilizados contatos pessoais e as redes sociais de forma pública como Instagram ${ }^{\circledR}$, Facebook ${ }^{\circledast}$ e Whatsapp ${ }^{\circledast}$ como disseminadores do questionário. 0 instrumento ficou disponivel durante uma semana entre os dias 20 e 27 de abril de 2020. Portanto, ela aconteceu durante o isolamento social em que os brasileiros de todos os estados deveriam permanecer em regime de quarentena em seus domicílios e com fechamento de todos os estabelecimentos que não fossem essenciais. A necessidade da observação da população nesse período ocorreu devido a possíveis mudanças de opinião decorrentes do período de confinamento, já que al guns realizavam quarentenas voluntárias outros não.

Para análise dos dados foi utilizado o banco de dados brutos gerados pelo Google Planilhas ${ }^{\circledR}$ e o relatório gerado pelo Typeform $^{\circledR}$ contendo os percentuais de respostas de cada questão. Foram construídas tabelas, onde se agrupou as respostas por variáveis às questões do tipo likert, sobre medos e preocupações, e apresentaram-se as frequências de respostas absolutas (N) e percentuais (\%) para apontar as principais preocupações e medos revelados pelas participantes, apresentadas por ordem decrescente de valor. Os questionários parcialmente respondidos foram excluídos do estudo.

\section{Resultados e discussão}

\section{Aspectos sociodemográficos}

Nessa seção, são apresentadas as variáveis: faixa etária, estado civil, cor da pele, nível de escolaridade, ocupação principal e renda, que caracterizam a amostra do ponto de vista sociodemográfico. Esses dados podem ser visualizados na Tabela 1.

Tabela 1: Caracterização sociodemográfica da amostra.

\begin{tabular}{|c|c|c|c|}
\hline \multicolumn{2}{|c|}{ Variáveis } & \multirow{2}{*}{$\frac{\mathbf{N}}{86}$} & \multirow{2}{*}{$\begin{array}{l}\% \\
8,2\end{array}$} \\
\hline Faixa etária & $18-24$ anos & & \\
\hline & $25-29$ anos & 02 & 19,3 \\
\hline & $30-34$ anos & 428 & 40,8 \\
\hline & $35-39$ anos & 292 & 27,8 \\
\hline & $40-44$ anos & 37 & 3,5 \\
\hline & 45 ou mais anos & 4 & 0.4 \\
\hline \multirow[t]{4}{*}{ Estado Civil } & Casada / União estável & 949 & 90,5 \\
\hline & Solteira & 82 & 7,8 \\
\hline & Separada/divorciada & 18 & 1,7 \\
\hline & Viúva & 0 & 0 \\
\hline \multirow[t]{5}{*}{ Cor da pele } & Branca & 709 & 67,6 \\
\hline & Parda & 256 & 24,4 \\
\hline & Preta & 53 & 5,05 \\
\hline & Amarela & 29 & 2,76 \\
\hline & Indígena & 2 & 1,19 \\
\hline \multirow[t]{6}{*}{ Escolaridade } & Ensino fundamental & 4 & 0,38 \\
\hline & Ensino médio incompleto & 10 & 0,95 \\
\hline & Ensino médio completo & 60 & 5,72 \\
\hline & Ensino superior incompleto & 96 & 9,15 \\
\hline & Ensino superior completo & 357 & 34,0 \\
\hline & Pós-graduação & 522 & 49,8 \\
\hline
\end{tabular}




\begin{tabular}{llll}
\hline Ocupação & Autônoma liberal & 312 & 29,7 \\
& Servidora pública & 261 & 24,9 \\
& Empregada (CLT) & 257 & 24,5 \\
& Do lar & 108 & 10,3 \\
& Desempregada & 57 & 5,4 \\
& Estudante & 54 & 5,1 \\
\hline Renda & Sem renda & 140 & 13,4 \\
& Até 1 SM & 82 & 7,8 \\
& De 1 1a 3 SM & 271 & 25,8 \\
& De 4a 7 SM & 254 & 24,2 \\
& De 8a 10 SM & 109 & 10,4 \\
& Acima de 10 SM & 158 & 15,1 \\
& Não sabe informar & 35 & 3,4 \\
\hline & & $\mathbf{1 0 4 9}$ & $\mathbf{1 0 0}$ \\
\hline
\end{tabular}

Fonte: dados da pesquisa.

Na Tabela 1, pode-se observar que das 1.049 gestantes e puérperas participantes a maior adesão foi de mulheres na faixa etária entre 30 e 34 anos ( $n=428$ ou $40,8 \%$ da amostra). A imensa maioria das participantes ( $n=949$ ou 90,5\%) estava casada ou em união estável; 709 ou 67,6\% das respondentes se declararam de raça branca; 830 ou $79,1 \%$ das mulheres exerciam atividade remunerada e 525 ou $50 \%$ delas tinha renda de um a sete salários mínimos. Quanto à escolarização, a grande maioria das respondentes afirmou possuir nível superior completo (n=357 ou 34\%) e 522 ou 49,9\% tinham pós-graduação. Esses resultados revelaram que a maioria da amostra possuía nível alto de escolaridade, estava trabalhando e tinha boas condiçoes de renda, representando, portanto, apenas parte da população de gestantes e puérperas brasileiras. Em função disso, os resultados devem ser relativizados enão podem ser generalizados para a população brasileira. Nossa amostra se assemelha em alguns aspectos e se diferencia em outros das amostras de pesquisas envolvendo gestantes e puérperas brasileiras. Cabe ressaltar que Dong et al. (2021) alertam que fatores que afetam o estado mental das gestantes são complexos e multifacetados e incluem idade, escolaridade, ocupação, semana gestacional, renda econômica, histórico médico, fatores sociopsicológicos, entre outros.

\section{Aspectos relacionados aos sentimentos de medo e de preocupação durante o isolamento social na pandemia}

Em relação às gestantes estudadas, os resultados mostraram que as suas principais preocupaçoes e temores apontavam para: ter a Covid-19 e ser internada numa UTI; o bebê precisar de UTI neonatal; ter a Covid-19 e perder 0 bebê, com índices de resposta de 88\%,86\% e 77\%, respectivamente. Outras preocupações e medos também apareceram com alta frequência nas respostas das gestantes: transmitir o coronavírus para o bebê ainda dentro do útero; 0 bebê adquirir uma má-formação em caso da mãe ter sido contaminada pelo coronavírus, com incidência de 73\% e 66\% cada. Na Tabela 2, esses dados podem ser visualizados. Na pesquisa de Puertas-Gonzalez et al. (2021), feita com gestantes espanholas, também foi identificado o medo de contágio pelo COVID-19, pelas gestantes o que os levou a concluir que a pandemia COVID-19 pode produzir um aumento na sintomatologia psicopatológica e no estresse, o que pode afetar negativamente a saúde mental das mulheres grávidas.

Tabela 2: Principais preocupações e medos das gestantes ( $n=710)$ frente à pandemia pelo coronavirus durante a gestação.

\begin{tabular}{lll}
\hline Preocupações e medos das gestantes & N & $\%$ \\
\hline Ter a Covid-19 e ser internada numa UTI & 625 & 88 \\
Bebê precisar de UTI neonatal & 611 & 86 \\
Ter a Covid-19 e perder o bebê & 547 & 77 \\
Ter um parto prematuro por causa da pandemia & 540 & 76 \\
Morrer na gestação por causa da Covid-19 & 540 & 76 \\
Transmissão vertical do coronavírus & 519 & 73 \\
Bebê adquirir uma má formação por causa do coronavírus & 469 & 66 \\
\hline
\end{tabular}

Fonte: dados da pesquisa.

Esses resultados revelam que parte dessas preocupações são esperadas, inerentes ao período gestacional (Schetter, 2011; Saviani-Zeoti, \& Petean,
2015). Sabe-se que a gravidez e a maternidade são períodos de mudança de vida caracterizados por emoções intensas e uma alta vulnerabilidade a 
problemas emocionais (Schetter, 2011; Saviani-Zeoti, \& Petean, 2015). № geral, 10\% a 20\% das mulheres grávidas e mulheres no período pós-parto precoce sofrem algum tipo/grau de adoecimento psíquico (Saviani-Zeoti, \& Petean, 2015). Portanto, nossos resultados revelam essas preocupações são naturais, inerentes ao período gestacional e até realistas frente à pandemia que estamos vivendo. Esses resultados são compatíveis com estudos que mostraram que as pandemias são precursoras de um aumento da sintomatologia psicopatológica e podem afetar a saúde mental de mulheres grávidas (Puertas-Gonzalez et al., (2021; Dong et al, 2021).

De fato, o medo de ser infectado pelo coronavírus e desenvolver a Covid-19 tem se configurado como a principal preocupação da população brasileira em geral (Schmidt et al., 2020). Diante de cenário de pandemia, a população tende a vivenciar agentes estressores relacionados ao risco de contrair ou de transmitir para outras pessoas, à sobrecarga de trabalho, à fadiga, às mortes em larga escala, ao afastamento da família e de pessoas queridas (Schmidt et al., 2020). Cabe ressaltar que em alguns casos, a incerteza sobre infecção e morte ou sobre infectar familiares e amigos pode potencializar estados mentais disfóricos (Xiang et al., 2020). Entretanto, parece que esses medos são potencializados pela gestação, uma vez que além do medo dela própria ser infectada com o vírus, há ainda o medo de que a infecção possa ser transmitida para o bebê que ela carrega em seu ventre (Knight et al., 2020; López-Morales et al., 2021).

Cabe ainda ressaltar que, para entender as repercussões psicológicas de uma pandemia, as emoções envolvidas, como medo e preocupações, devem ser consideradas e observadas. 0 medo é um mecanismo de defesa animal adaptável que é fundamental para a sobrevivência e envolve vários processos biológicos de preparação para uma resposta a eventos potencialmente ameaçadores. No entanto, quando é crônica ou desproporcional, torna-se prejudicial e pode ser um gatilho ou catalisador para o desenvolvimento de vários transtornos psiquiátricos (Xiang et al., 2020). Em uma pandemia, 0 medo aumenta os níveis de ansiedade e estresse em indivíduos saudáveis e intensifica os sintomas daqueles com transtornos psiquiátricos existentes (Fiocruz., 2020; Ornell et al., 2020).

Os resultados do presente estudo são concordes com uma pesquisa feita na Turquia por Sahina e Kabakci (2021) que mostram que a pandemia de coronavírus tem um potencial significativo para criar ansiedade, adversidade e medo, o que tem um efeito emocional negativo nas mulheres grávidas. Por isso, assim como esses autores, nós também recomendamos que os profissionais da obstetrícia não cuidem apenas da saúde física das mulheres grávidas, mas também sobre sua saúde mental, e incluir especialistas em saúde mental nesses cuidados.

De acordo com a The Marcé Society (2020), sabe-se que a presença de qualquer situação que ponha em risco a vida humana ativa um conjunto de preocupações, sobretudo em mães e pais durante a gravidez e/ou na prestação de cuidados a o bebê. A dificuldade em lidar com a adversidade associada a esse tipo de situação, à pandemia soma-se um conjunto de restrições que podem ser necessárias e que obrigam a alterar os cuidados e a relação com o bebê, em particular se um ou ambos os pais estiverem com a Covid-19. Ter que lidar com as adversidades e riscos inerentes à doença, reorganizar práticas de cuidados parentais e ainda dar conta das demandas de trabalho e tarefas domésticas, durante uma pandemia, pode ser particularmente difícil para os pais e acarretar em adoecimento psíquico (WHO, 2019b; López-Morales et al. ,2021).

Porém, chamou atenção das pesquisadoras, os altos índices de preocupações relacionadas à transmissão vertical do coronavírus e ao bebê adquirir uma má-formação por causa desse vírus. Esses medos que parecerem povoar 0 imaginário das gestantes, não tem respaldo na realidade, pois até 0 momento não foi descrito qualquer um desses casos nos bebês, mesmo entre as gestantes que testaram positivo para o coronavírus e/ou que manifestaram a Covid-19. Por outro lado, ainda que ainda não existem dados suficientes sugerindo um risco aumentado de anomalias congênitas, aborto espontâneo, restrição do crescimento intrauterino, entre outros desfechos relacionados à Covid-19 (Yang et al., 2019; Takemoto et al., 2020), a literatura é mais do que evidente quanto ao impacto das epidemias, catástrofes naturais, guerras e outras situações que põem em risco ou ameaçam a vida, na saúde (mental), sobretudo das mães e pais no período perinatal, e por consequência na saúde do feto/bebês, conduzindo ao aumento da incidência, entre outras, de aborto espontâneo e parto prematuro (Seng et al., 2001), bem como ao impacto da saúde mental na resolução dos estados de infecção (Ceulemans, Hompes, \& Foulon, 2020; Saviani-Zeoti, \& Petean, 2015; Puertas-Gonzalez et al., 2021).

Da mesma forma, quando pensam no trabalho de parto e parto, as gestantes continuam a nutrir preocupações com a possibilidade de elas próprias serem infectadas ou de seus bebês contraírem o coronavírus durante 0 nascimento, com frequência de $75 \%$ e $78 \%$ das respostas. Esses dados podem ser observados na Tabela 3.

Tabela 3: Principais preocupações/medos das gestantes $(n=710)$ frente à pandemia pelo coronavírus, durante 0 trabalho de parto e parto

\begin{tabular}{lll}
\hline Preocupações/medos das gestantes & N & $\%$ \\
\hline Não poder ter acompanhante na sala de parto & 561 & 79 \\
Bebê se contaminar com o coronavírus durante o parto & 554 & 78 \\
Ser contaminada com o coronavírus durante o parto & 532 & 75 \\
Não ser respeitada durante o trabalho de parto e parto & 504 & 71 \\
Não poder fazer pele a pele & 497 & 70 \\
Sofrer violência obstétrica justificada pela pandemia no trabalho de & 490 & 69 \\
$\qquad$ parto e parto & & \\
Falta de vaga na maternidade & 447 & 63 \\
Não poder seguir o plano de parto & 376 & 53 \\
\hline
\end{tabular}

Fonte: dados da pesquisa. 
Observa-se na Tabela 3, que a grande maioria das preocuações apresentadas tem respaldo não só na realidade, mas também nos estudos apresentados até o momento. De fato, encontrou-se que, tanto mães quanto profissionais ou acompanhantes presentes na sala de parto que estejam contaminadas com o coronavírus, mesmo assintomáticos ou com a Covid-19, podem ter contaminado os bebês durante ou logo após o nascimento (Frota et al., 2020). Talvez por mesma essa razão, chamou atenção das pesquisadoras que a maior preocupação apresentada pelas gestantes foi "Não poder ter acompanhante na sala de parto", com 79\% das respostas.

Entretanto, ter uma acompanhante durante o parto é um direito garantido pela legislação (Lei Federal nº 11.108/2005) que não foi revogado até 0 momento. Ainda que várias restrições nos ambientes hospitalares obstétricos tenham sido impostas desde a implementação do isolamento social, ocorrido em meados de março de 2020 em várias cidades do Brasil, como a proibição de visitas e diminuição de profissionais circulando na sala de parto, a presença do acompanhante na cena do parto, a princípio continua sendo um direito garantido, mesmo em tempos de pandemia.

No caso dos acompanhantes, a recomendação é apenas que caso esteja sintomático, seja substituído por uma/um acompanhante da escolha da parturiente que esteja sem sintomas de Covid-19. Por que mesmo com a garantia da lei, 79\% das gestantes respondentes ainda apresentam essa grande preocupação? Interessante ressaltar também que a essa mesma preocupação aparece em 10 lugar no pós-parto, como pode ser visualizado na Tabela 4, a seguir.

Ainda fazem parte das preocupações das gestantes, que aparecem na Tabela 3, não ser respeitada durante 0 trabalho de parto e parto para 71\% delas; não poder fazer o pele a pele para 70\%; falta de vaga na maternidade, para 63\%; não poder seguir o plano de parto, para 53\% das gestantes participantes. Não ser acompanhada por obstetras, seja médico ou enfermeiro, para 47\% dessas mulheres.

Tabela 4: Principais preocupações das gestantes $(n=710)$ frente à pandemia pelo coronavírus, durante o pós-parto imediato e tardio.

\begin{tabular}{lll}
\hline Preocupações das gestantes & N & $\%$ \\
\hline Ficar sem acompanhante no pós-parto imediato & 625 & 88 \\
Não ser respeitada quanto às escolhas e desejos no pós-parto & 532 & 75 \\
$\quad$ imediato & & \\
Sofrer violência obstétrica justificada pela pandemia no pós-parto & 504 & 71 \\
imediato & 490 & 69 \\
Não poder amamentar o bebê se sintomática e/ou com & & \\
a Covid-19 & 483 & 68 \\
Morrer no pós-parto por causa da Covid-19 & 461 & 65 \\
Medo de ou ficar sozinha & 461 & 65 \\
Não poder contar com a ajuda da minha mãe/família & 454 & 64 \\
Não poder amamentar o bebê mesmo sem Covid-19 & \\
\hline
\end{tabular}

Fonte: dados da pesquisa.

Os dados da Tabela 4 mostram que quando pensavam no pós-parto imediato e tardio, 69\% das gestantes se preocupavam em não poder amamentar seus bebês ao seio por causa da apresentação de sintomas ou diagnóstico de Covid-19, sendo que índice semelhante ou 64\% das gestantes também apresentaram preocupação em não poder amamentar mesmo que não tivessem sintomas. Portanto, não amamentar o bebê por causa da pandemia é uma grande preocupação das gestantes, tendo ou não sintomas ou confirmação de terem sido contaminadas pelo coronavírus.

Mais uma vez, as pesquisadoras observaram a presença de preocupações e temores que não têm respaldo na realidade e que não são corroborados por orientações oficiais e com as evidências científicas disponíveis até o momento (The MarcéSociety, 2020). Por isso, são necessárias e importantes todas as ações que possibilitem reduzir o impacto da restrição dos cuidados e contatos da mãe/ pai sinalizados ou confirmados com infecção pelo coronavírus ou com a Covid-19, nomeadamente no que se refere à amamentação e à relação com o bebê, imprescindíveis à saúde mental da mãe, do pai e do bebê (Xiang et al., 2020).

Sobre o pós-parto imediato, 75\% das gestantes temiam não serem respeitadas em suas escolhas e desejos. Além disso, ficar sozinha ou não poder contar com a ajuda da mãe ou da família era uma preocupação para 65\% dessas mulheres. Em contrapartida, não poder receber visitas em casa no pós-parto imediato era uma preocupação para, 34\% das gestantes que participaram da pesquisa. Ter apoio no pós-parto é fundamental para a prevenção de adoecimentos e sofrimentos psíquicos, além de serem aliados para a constituição do papel materno.

0 medo de morrer no pós-parto é um medo frequente em gestantes e estão relacionados ao abandono do bebê e às crenças sociais negativas que as mães são as que sabem cuidar melhor de seus bebês e que são as únicas que serão capazes de nutrir um amor incondicional pela criança (Schetter, 2011; Saviani-Zeoti \& Petean, 2015). A pandemia pode ter intensificado esse medo, dando mais contornos a esse fantasma. Ademais, além de um medo concreto da morte, a pandemia do Covid-19 tem implicações para outras esferas: organização familiar, fechamento de escolas, empresas e locais públicos, mudanças nas rotinas de trabalho, isolamento, levando a sentimentos de desamparo e abandono. Pode ainda, aumentar a insegurança devido às repercussões econômicas e sociais dessa tragédia em larga escala (Ornell et al., 2020). 
A pesquisa também procurou saber quais as preocupações das mães de bebês (puérperas) nascidos durante a pandemia, com relação aos períodos de pós-parto imediato e tardio. Responderam à pesquisa 339 puérperas, que apresentaram as preocupações listadas na Tabela 5.

Tabela 5: Principais preocupações das puérperas ( $n=339)$ frente à pandemia pelo coronavírus, durante o pós-parto imediato e tardio.

\begin{tabular}{lll}
\hline Preocupações das puérperas & N & $\%$ \\
\hline Ter alguma emergência com bebêe e não poder levá-lo ao hospital & 271 & 80 \\
Bebê ser contaminado por alguém que esteja infectado & 247 & 73 \\
Não poder contar com a ajuda da minha mãe/família & 213 & 63 \\
Não poder levar o bebê para as consultas de rotina por conta do & 203 & 60 \\
isolamento social & & \\
Não poder amamentar o bebê se sintomática e/ou com a Covid-19 & 197 & 58 \\
Não ter todo suporte de profissionais por conta do isolamento social & 190 & 56 \\
Medo de ou ficar sozinha & 186 & 55 \\
Estar em contato com pessoas que não estão cumprindo o isolamento & 186 & 55 \\
social & & \\
Não poder receber as visitas em casa no pós-parto & 163 & 48 \\
Contar apenas com pai nos cuidados com o bebê & 159 & 47 \\
\hline
\end{tabular}

Fonte: dados da pesquisa.

Observa-se que $80 \%$ das respostas revelam que a maior preocupação das puérperas era não poder levar o bebê ao hospital, em caso de alguma emergência - seja pelas medidas de isolamento social, pelo risco de contaminação, pela falta de vagas no hospital. Interessante ressaltar que esse temor não apareceu como uma preocupação para as gestantes quanto ao período do pós-parto. 0 risco de o bebê ser contaminado por al guém que esteja infectado pelo coronavírus aparece em 20 lugar com $73 \%$ das respostas, e vai ao encontro das principais preocupações das gestantes. Não poder amamentar ao seio 0 bebê se a mãe apresentar sintomas da Covid-19 é uma grande preocupação para 58\% das puérperas, embora não figure entre os maiores temores. Mais uma vez, observa-se um resultado semelhante aos temores das gestantes, ainda que em um percentual menor, que foi de 64\%. Chama atenção que mesmo com índice menor, as puérperas também parecem apresentar as mesmas fantasias e crenças em relação a não possibilidade de amamentação que as gestantes.

São preocupações menores, porém ainda assim importantes, para $56 \%$ das respondentes não ter todo suporte de profissionais por conta do isolamento social; para 55\% das respondentes ficar sozinhas com o bebê; e também para 55\% entrar em contato com pessoas que não estão cumprindo 0 isolamento social.

Cabe ressaltar que em meados de abril de 2020, 0 Ministério da Saúde incluiu todas as gestantes e mães de bebês de até 60 dias no grupo de risco para a Covid-19 (Brasil, 2020c) . Atéo presente momento, como orientado pela Sociedade Brasileira de Pediatria (SBP, 2020), deve-se continuar estimulando a amamentação ao seio, com os devidos cuidados pela mãe, especialmente 0 uso de máscara durante a amamentação, se ela estiver infectada e/ou sintomática, Até agora, se as medidas de prevenção forem adotadas, as pesquisas não apontam para a presença de coronavírus no leite materno, no líquido amniótico, no sangue do cordão umbilical ou em secreções vaginais (Ceulemans, Hompes, \& Foulon, 2020).
É importante salientar que nesta pesquisa, 49\% das respondentes afirmaram possuir pós-graduação e 34\% ensino superior. Além disso, a maioria absoluta das mulheres afirmou que buscava informações sobre a pandemia em órgãos oficiais nacionais, como o Ministério da Saúde, e internacionais, como a OMS. Porém, como afirma a Fiocruz (2020), o desencontro de informaçõese as informações contraditórias emitidas por órgãos oficiais nacionais, como 0 Ministério da Saúde e a Presidência da República, podem gerar a sensação de insegurança nas informações obtidas, junto ao fato de que, em um momento como o de uma pandemia, as pesquisas não são produzidas em larga escala e vão acontecendo conforme vão surgindo os casos (Fiocruz, 2020).

Ficar sozinha/medo do desamparo, foi objeto de um estudo chinês que forneceu algumas ideias a respeito. Aproximadamente metade dos entrevistados classificou o impacto psicológico da epidemia como moderado a gravee cerca de um terço relatou ansiedade moderada a grave (Wang, C. et al., 2020; Ornell et al., 2020). Essas condições podem ser especialmente prevalentes em gestantes e puérperas em quarentena, cujos problemas psicológicos e 0 sofrimento tendem a ser maiores (Xiang et al., 2020). Segundo Dong et al. (2021) durante a epidemia, a saúde mental das mulheres grávidas está mais vulnerável do que o normal. A saúde psicológica da gestante é de extrema importância e deve ser atentada a qualquer momento, não apenas durante a epidemia.

\section{Repercussões práticas da pesquisa}

Os resultados da presente pesquisa motivaram a criação, pelas pesquisadoras, de um grupo voluntário formado por 21 psicólogos perinatais, que foi intitulado de Grupo de Intervenção em Crise (GIC), para oferecer atendimento on line às gestantes e puérperas durante a pandemia. Esse grupo foi criado para que possa subsidiar as estratégias e dar suporte psicossocial no atendimento dos profissionais da psicologia (The Marcé Society, 2020), as gestantes e puérperas em sofrimento pela pandemia de Covid-19. 0 GIG 
tem se disponibilizado para atendimento às Gestantes e Puérperas que estão em sofrimento na pandemia do Covid-19. A recomendação atual é que a intervenção deve ser uma intervenção psicológica especializada, dinâmica e flexível (Schmidt et al., 2020), o suficiente para se adaptar mais facilmente às diferentes fases da pandemia (Fiocruz, 2020; Xiang et al., 2020).

Da mesma forma, éimportante considerar que as autoridades de saúde, nos níveis nacional e regional, devem considerar equipes multidisciplinares de saúde mental, incluindo psiquiatras, psicólogos e outros profissionais de saúde mental, para trabalhar em sua abordagem. Responder às preocupações e oferecer estratégias para aumentar a sensação de segurança e controle é um objetivo da intervenção que propomos no sentido de manter a saúde mental e bem-estar dos pais. Diminuir o isolamento e aumentar o suporte social, emocional e instrumental, é também um objetivo para prevenir a sintomatologia ansiosa e depressiva dos pais (Schmidt et al., 2020). Ajudar os pais a estabelecer a relação com o bebê e a tomar decisões no que se refere aos cuidados com ele, no quadro da situação de distanciamento específica em que cada um ou ambos se encontram, também é objetivo do GIG e se baseiam nas recomendações da The Marcé Society (2020).

É importante destacar, ainda, que qualquer intervenção deve basear-se anteriormente em uma avaliação exaustiva dos prováveis fatores de risco que podem estar relacionados ao problema, como histórico de saúde mental do paciente, luto anterior, autoagressão ou comportamentos suicidas anteriores que ocorreram no paciente ou em sua família, além de histórico de trauma anterior, bem como seu contexto socioeconômico (Fiocruz, 2020; Schmidt et al., 2020)..

Especificamente para esse novo cenário da Covid-19, Xiang et al. (2020) sugerem que três fatores principais sejam considerados ao desenvolver estratégias de saúde mental: a) equipes multidisciplinares de saúde mental (incluindo psiquiatras, enfermeiros psiquiátricos, psicólogos e outros profissionais de saúde mental); b) comunicação clara envolvendo atualizações regulares e precisas sobre o surto de (ovid-19; e c) estabelecimento de serviços seguros de aconselhamento psicológico (por exemplo, por meio de dispositivos ou aplicativos eletrônicos). (abe ressaltar que essa iniciativa de disponibilizar 0 GIG, vai ao encontro dessas recomendações estratégicas, que também foram reforçadas pela Fiocruz (2020) e pela The Marcé Society (2020).

\section{Considerações finais}

Diante dos resultados, sugerimos a importância de estabelecer políticas preventivas focadas na saúde mental materna durante a pandemia, dirigidas ou desenvolvidas por profissionais de saúde mental (psicólogos clínicos e da saúde, psiquiatras, e especialmente psicólogos, perinatais) (Schmidt et al., 2020; Duan et al., 2020; Puertas-Gonzalez et al., 2021). É fundamental 0 desenvolvimento de ações de saúde voltadas para as demandas psicossociais do ciclo gravídico puerperal, sugerindo que os grupos de gestantes e puérperas devem ser identificados e adaptados às diferentes fases da pandemia, das regiões do Brasil e as diferentes realidades brasileiras. Ressalta-se a importância do acolhimento ao sofrimento psíquico dessa clientela, da educação e fornecimento de informações atualizadas e de qualidade pelas agências governamentais, para bem enfrentar o momento atual (Duan et al., 2020).

Por fim, é extremamente necessário implementar políticas públicas de saúde mental em conjunto com estratégias de resposta à epidemia e pandemia antes, durante e após o evento. Profissionais de saúde mental, como psicólogos, psiquiatras e assistentes sociais, devem estar na linha de frente e brincar um papel de liderança nas equipes de planejamento e gerenciamento de emergências (Ornell et al., 2020; Sahina, \& Kabakci, 2021). É urgente e necessário um aumento do investimento em pesquisas e ações estratégicas para a saúde mental em paralelo com surtos infecciosos (The Marcé Society 2020; Ornell et al., 2020; Duan et al., 2020).

\section{Referências}

Brasil (2020a). Ministério da Saúde Brasil confirma primeiro caso da doença. Disponível em: https://www.gov.br/pt-br/noticias/saude-e-vigilancia-sanitaria/2020/02/brasil-confirma-primeiro-caso-do-novo-coronavirus

Brasil (2020b). Ministério da Saúde Secretaria de Vigilância em Saúde. Centro de Operações de Emergências em Saúde Pública (COE-nCOv). Infecção Humana pelo Novo Coronavirus (2019-nCoV). Ministério da Saúde - Brasília.

Brasil (2020c). Ministério da Saúde Protocolo de Manejo Clínico da Covid-19 na Atenção Especializada. p. 05.

Croda, J. H. Rosa e Garcia, L. P. Resposta imediata da Vigilância em Saúde à epidemia da COVID-19. Epidemiologia e Serviços de Saúde [online]. 2020, v. 29, n. 1. Doi.org/10.5123/S1679-49742020000100021

Ceulemans, M., Hompes, T., \& Foulon, V. (2020). Estado de saúde mental de mulheres grávidas e que amamentam durante a pandemia do COVID-19: um apelo à ação. International Journal of Gynecology \& Obstetrics, 151(1), 146-147. Doi:10.1002/ijgo.13295

Dong, H., Hu, R., Lu, C. etal. (2021). Investigation on the mental health status of pregnant women in China during the Pandemic of COVID-19. Arch Gynecol Obstet 303, 463-469. https://doi.org/10.1007/s00404-020-05805-x

Duan, L., \& Zhu, G. (2020). Psychological interventions for people affected by the Covid-19 epidemic. The Lancet Psychiatry, 7(4), 300-302. doi:10.1016/ S2215-0366(20)30073-0

Favre, G., Pomar, L., Musso, D., \& Baud, D. (2020). 2019-nCoV epidemic: what about pregnancies? Lancet, 395(10224), E40. doi:10.1016/S01406736(20)30311-1.

Fiocruz. (2020). Saúde Mental e Atenção Psicossocial na Pandemia Covid-19 - Recomendações Gerais. Disponivel em: https://www.fiocruzbrasilia. fiocruz.br/wp-content/uploads/2020/04/Sa\%c3\%bade-Mental-e-Aten\%c3\%a7\%c3\%a30-Psicossocial-na-Pandemia-Covid-19-recomenda\%c3\%a7\%c3\%b5es-gerais.pdf

Filho, H.R.C., \&Segall-Corrêa, A.M. (2020). Lockdown ou vigilância participativa em saúde? Lições da Covid-19. Saúde Debate, 44(124):5-16.

Frota et al. (2020). Assistência Obstétrica à Portadora de COVID-19: uma revisão integrativa/ Obstetric Assistance to the Bearer of COVID-19: an integrative review. Braz. J. Hea. Rev, Curitiba, 3 (6): 18342-18358. doi: https://doi.org/10.34119/bjhrv3n6-232

Huang, C. et al. (2020). Clinical features of patients infected with 2019 novel coronavirus in Wuhan, China. Lancet, 95(10223):497-506.

Kang, L. et al. (2020). The mental health of medical workers in Wuhan, China dealing with the 2019 novel coronavirus. The Lancet Psychiatry, 7(3), E14. doi:10.1016/S2215-0366(20)30047-X

Knight M, Bunch K, Vousden N, et al. (2020). Characteristics and outcomes of pregnant women admitted to hospital with confirmed SARS-COV-2 infection in UK: national population based cohort study. BMJ, 369, m2107. Doi:10.1136/bmj.m2107

López-Morales et al. (2021). Mental health of pregnant women during the COVID-19 pandemic: A longitudinal study. Psychiatry Research, 295, 113567. https://doi.org/10.1016/j.psychres.2020.113567

Ornell, F., Schuch, J.B., Sordi, A.O., \& Kessler, F.H.P. (2020). Pandemic fear" and Covid-19: mental health burden and strategies. Braz J Psychiatry, 42(3), 232-235. doi:10.1590/1516-4446-2020-0008 
Parmet, W.E., \& Sinha, M.S.S. (2020). Covid-19 — The Law and Limits of Quarantine. N Engl J Med., 382(15): e28. doi: 10.1056/NEJMp2004211

Puertas-Gonzalez et al. (2021). The psychological impact of the COVID-19 pandemic on pregnant women. Psychiatry Research, 301, 113978. doi. org/10.1016/j.psychres.2021.113978

Sahina, B.M., \& Kabakci, E.N. (2021). The experiences of pregnant women during the COVID-19 pandemic in Turkey: A qualitative study. Women and Birth, 34 (2): 162-169. doi.org/10.1016/j.wombi.2020.09.022

Saviani-Zeoti, F., \& Petean, E.B.L. (2015). Apego materno-fetal, ansiedade e depressão em gestantes com gravidez normal e de risco: estudo comparativo. Estud. psicol. (Campinas), 32(4),675-683. doi:10.1590/ 0103-166X2015000400010.

Sarti, T.D., Lazarini, W.S., Fontenelle, L.F., \& Almeida, A.P.S.C. (2020). Qual 0 papel da Atenção Primária à Saúde diante da pandemia provocada pela Covid-19?. Epidemiol. Serv. Saude, 29(2): e2020166.

Schetter, C.D. (2011) Psychological Science on Pregnancy: Stress Processes, Biopsychosocial Models, and Emerging Research Issues. Annu. Rev. Psychol., 62:531-58, 2011. doi: 10.1146 / annurev.psych.031809.130727.

Schmidt, B., Crepaldi, M.A., Bolze, D.A.S., Neiva-Silva, L., \& Demenech, L.M. (2020). Saúde mental e intervenções psicológicas diante da pandemia do novo coronavírus (Covid-19). Estudos de Psicologia (Campinas), 37,e200063. doi:10.1590/1982-0275202037e200063

Seng, J.S., Oakley, D.J., Sampselle, C.M., Killion, C., Graham-Bermann, S., \& Liberzon, I. (2001). Posttraumatic stress disorder and pregnancy complications. Obstetrics \& Gynecology, 97(1), 17-22. doi:10.1016/ S0029-7844(00)01097-8

Sociedade Brasileira de Pediatria (SBP). (2020). Notade Alerta - Oaleitamento materno nos tempos de Covid-19. Disponível em: https://www.sbp.com. br/fileadmin/user_upload/22393c-Nota_de_Alerta_sobe_Aleitam_Materno_nos_Tempos_COVID-19.pdf

Takemoto, M.L.S., Menezes, M.O., Andreucci, C.B., Nakamura-Pereira, M., Amorim, M.M.R., Katz, L., \& Knobel, R. (2020). A tragédia da COVID囚19 no Brasil: 124 mortes maternas e contando. Internacional Journal of Gynecology \& Obstetrics, 151(1), 154-156. doi:10.1002/ijgo.13300

The Marcé Society. (2020). Quando a vida não escolhe tempo para nascer (VIDA). Recomendações do Grupo de Língua Portuguesa da Sociedade Marcé Internacional para a Saúde Mental Perinatal. Disponível em https:// marcesociety.com/covid-19-perinatal-mental-health-resources/

Xiang, YT. et al. (2020). Timely mental health care for the 2019 novel coronavirus outbreak is urgently needed. Lancet Psychiatry, 7: 228-9.

$X u$, Z. et al. (2020).Pathological findings of Covid-19 associated with acute respiratory distress syndrome. Lancet Respir Med., 8(4):420-422. doi: 10.1016/52213-2600(20)30076-X.

Wang, C. et al. (2020).Immediate psychological responses and associated factors during the initial stage of the 2019 coronavirus disease (Covid-19) epidemic among the general population in China. Int I Environ Res Public Health., 17(5):1729. doi: 10.3390/ijerph17051729.

World Health Organization (WHO) (2019a). Report of the WHO-China Joint Mission on Coronavirus Disease (COVID-19). Disponível em https://www. who.int/docs/default-source/coronaviruse/who-china-joint-mission-on-covid-19-final-report.pdf

World Health Organization (WHO). (2019b). Q\&A on Covid-19, pregnancy, childbirth and breastfeeding. Disponível em: who.int/news-room/q-a-detail/q-a-on-covid-19-pregnancy-childbirthand-breastfeeding

Yang, Z., Wang, M., Zhu, Z., \& Liu, Y. (2020). Coronavirus disease 2019 (COVID-19) and pregnancy: a systematic review. J Matern Fetal Neonatal Med, Apr 30,1-4. doi: 10.1080/14767058.2020.1759541. Epub ahead of print. PMID: 32354293. 